\title{
Extraction of Copper from Waste Solution Using Liquid Emulsion Membrane
}

\author{
Yasser T. Mohamed, Ahmed H. Ibrahim
}

Hot Laboratories and Waste Management Center, Atomic Energy Authority, Cairo, Egypt.

Email: ytselim@yahoo.com

Received June $9^{\text {th }}, 2011$; revised November $9^{\text {th }}, 2011$; accepted December $9^{\text {th }}, 2011$

\begin{abstract}
The extraction of copper from acidic medium using Liquid Emulsion Membrane LEM as contactors was studied. $\beta$ diketone LIX 54 as an extractant, sesqueoleate which is nonionic surfactant with HLB value 3.7 and $0.1 \mathrm{M} \mathrm{H}_{2} \mathrm{SO}_{4}$ as stripping solution were used. The influence of initial concentration of copper in the aqueous feed phase, $\mathrm{pH}$ of external and internal aqueous phase, stirring speed, and concentration of extractant on the extraction rate was examined. The results obtained showed that practically all the copper content was removed from the acidic feed solution. The recovery of copper attained $96 \%-100 \%$ and concentration ratios of about 40 -fold could be achieved.
\end{abstract}

Keywords: LIX 54; Copper Extraction; Liquid Emulsion Membrane

\section{Introduction}

There is a general concern to minimize the discharge of hazardous metals in waste streams. Aqueous waste streams contaminated with heavy metal ions are frequently encountered from various industrial processes such as mining and smelting, metal plating, metal finishing also during attempts to remediate soil loaded with heavy metals. Precipitation technique is almost commonly used for removal of metal species from waste streams. This technique is becoming unpopular because the products obtained are not always of required quality and the process produces a sludge that has to be disposed of in a landfill. Solvent extraction is another widely used technique, but it requires large amount of organic solvent and metal extractant and becomes inefficient when the metal ion concentration in the waste stream is low.

Liquid Emulsion Membrane (LEM) first invented by Li [1] offers a promising technology for the separation of heavy metal ions from aqueous solution. LEM removes the equilibrium limitation of solvent extraction by combining the extraction and stripping simultaneously in the same time thereby achieving reduction of metal concentration in the feed solution to a very low level. Furthermore, they reduce the amount of organic solvent and metal extractant substantially. LEMs have been used to treat aqueous waste contaminated with heavy metals like copper, zinc, cadmium, chromium, etc. [2-4]. LEMs for metal extraction are made by forming a water in oil (W/O) emulsion, stabilized by a surfactant, the W/O emulsion contains the metal extractant (carrier) in the oil phase and the stripping solution in the internal aqueous phase. This emulsion is then dispersed by mild agitation into a feed solution containing the metal ions to be extracted. After extraction, the loaded emulsion is separated from the feed solution, demulsification yields an oil phase that can be recycled. Electroplating or crystallization could subsequently recover the heavy metal concentrated in the stripping phase. Removal of copper as $\mathrm{Cu}^{2+}$ ions from aqueous waste solutions using LEMs has been an area of interest ever since LEMs were invented. Most investigators [5-8] preferred using chelating extractants, such as LIX 63, LIX 64N, LIX 65, SME 529 ${ }^{\circledR}$, etc., because of their immiscibility with water. Economic evaluion by Frankenfeld [9] showed that LEM extraction of copper using LIX $64 \mathrm{~N}$ turns out to be $40 \%$ cheaper than solvent extraction. Earlier used copper extractants LIX 63, LIX 65 , etc. are no longer commercially available. [10-12] investigated copper extraction into LEMs using the widely used extractant LIX 84 as carrier. Cognis Inc. has introduced new copper extractants such as LIX 54, LIX 860 and LIX 984 and their variants that show faster kinetics, easier phase disengagement and strong extraction of copper even at low $\mathrm{pH}$ values of 1 to 2 . LIX 54 is particularly attractive for extraction of copper since it combines the most desirable features of its components. The extractive strength and fast kinetics of $\beta$-diketone are combined with proven excellent physical performance and stability of $\beta$-diketone. In the present work, the extraction of copper from aqueous acidic medium into LEMs using LIX 54 as carrier is studied. The results 
present an overall idea of the optimal operating conditions for copper extraction into LEMs [13-15].

\section{Experimental}

\subsection{Chemicals and Reagents}

The extractant LIX54 is supplied by Cognis (Ireland), toluene and surfactants (span80, sesquoleate and span85) are supplied from Fluka AG. The other chemicals and reagents used were of A.R. grade.

\subsection{Membrane Preparation}

Unless otherwise stated, the LEM used is prepared by mixing $25 \mathrm{~cm}^{3}$ of the organic phase which containing, $15 \%$ LIX 54 with $8 \%$ sesquioleate as a surfactant in toluene. To this mixture $25 \mathrm{~cm}^{3}$ of the stripping aqueous phase solution (0.1 Sulfuric acid), was added. The emulsification was performed with an ultra high-speed homogenizer IKA, Ultra-Turrax T25, (USA). The rate of emulsion stratification, without contacting the feed solution was $0.15 \%$ per hour Figure 1. The water in oil (W/O) emulsion was then poured into the external aqueous phase containing $50 \mathrm{mg} / \mathrm{l} \mathrm{Cu}$ ions. The LEM system was stirred with a magnetic stirrer of the type Cole palmer (USA) of variable speed ranging from 150 to $750 \mathrm{rpm}$. Samples were withdrawn from the external phase, and the $\mathrm{Cu}$ concentration was analyzed by Atomic Absorption spectrometry, Model AA-6701F Shimadzu, Kyoto (Japan). The permeation percent $(\% \mathrm{P})$ of $\mathrm{Cu}$ through LEM was calculated by:

$$
\% \mathrm{P}=\left[\left(\mathrm{C}_{\mathrm{o}}-\mathrm{C}_{\mathrm{t}}\right) / \mathrm{C}_{\mathrm{o}}\right] \times 100
$$

where $\mathrm{C}_{\mathrm{o}}$ is the initial concentration of metal ions in the feed solution and $\mathrm{C}_{\mathrm{t}}$ is its concentration in the feed solution after time interval $t$. The stability of the prepared LEM was studied in terms of the degree of leakage of a Rohdamine dye as a tracer from the stripping solution to the feed solution by the following expression,

$$
\% \mathrm{~B}=\left([\text { dye }]_{\mathrm{ex}, \mathrm{t}} /[\text { dye }]_{\mathrm{in}, \max }\right) \times 100
$$

where $\mathrm{B}$ is the percent of emulsion breakage and [dye $]_{\mathrm{ex}, \mathrm{t}}$ is the concentration of dye leaked from internal aqueous

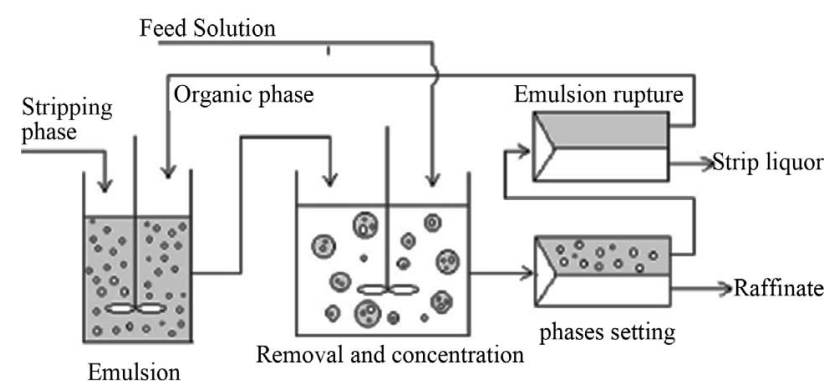

Figure 1. Schematic diagram of double emulsion liquid membrane process. phase to external aqueous phase and $[d y e]_{\text {in,max }}$ represents the maximum concentration of dye in external aqueous phase when all dye leaked from internal aqueous phase to external aqueous phase.

Unless otherwise stated the concentration of sesq- uioleate (HLB value 3.7) was $8 \% \mathrm{v} / \mathrm{v}$, stirring speed 450 rpm, the temperature was $298 \mathrm{~K}$ and the initial concentration of $\mathrm{Cu}$ in the internal aqueous phase was zero.

\subsection{Procedure}

Batch liquid-liquid extraction experiments were carried out by mixing equal volumes of the organic phase and the aqueous phase $\left(5 \mathrm{~cm}^{3}\right.$ each) containing copper ions of concentration of $100 \mathrm{mg} / \mathrm{l}$ unless otherwise stated. Equilibrium was attained by shaking the two phases for 30 minutes using a mechanical shaker. Known sample portions from aqueous phase were taken for metal ions determination before and after equilibrium to determine the extraction percent in the organic phase.

\section{Results and Discussion}

\subsection{Liquid-Liquid Extraction}

Extraction equilibrium was first examined to identify the extraction behavior and the suitable conditions for $\mathrm{Cu}$ extraction from acidic medium using LIX 54 as a carrier and toluene as a diluent. The effect of $\mathrm{pH}$ on the extraction percent of $\mathrm{Cu}$ showed that the extraction percent increases with increasing the $\mathrm{pH}$ of aqueous phase, the optimum $\mathrm{pH}$ is 4.3 at which the $\mathrm{CuSO}_{4}$ started to be disappeared and converted to $\mathrm{Cu}_{3} \mathrm{SO}_{4}(\mathrm{OH})_{4}$ complex Figure 2. The effect of LIX 54 concentration on the extraction percent of $\mathrm{Cu}$ at $\mathrm{pH} 4.3$ showed that, the extraction percent increases with increasing the LIX54 concentration Figure 3.

Aqueous solutions of $\mathrm{H}_{2} \mathrm{SO}_{4}, \mathrm{HCl}, \mathrm{NaOH}$, oxalic acid, Sodium acetate and sodium citrate were examined as

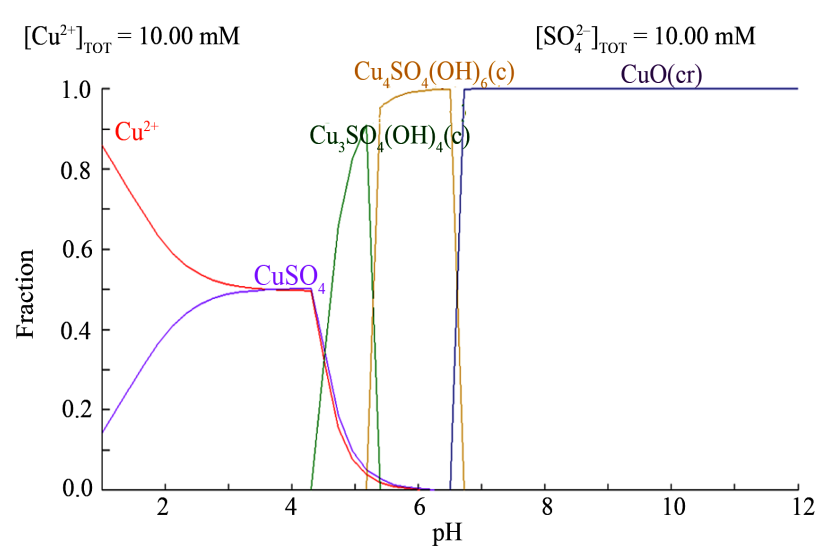

Figure 2. Effect of pH on the complex formation and ion species in the stirred tank. 


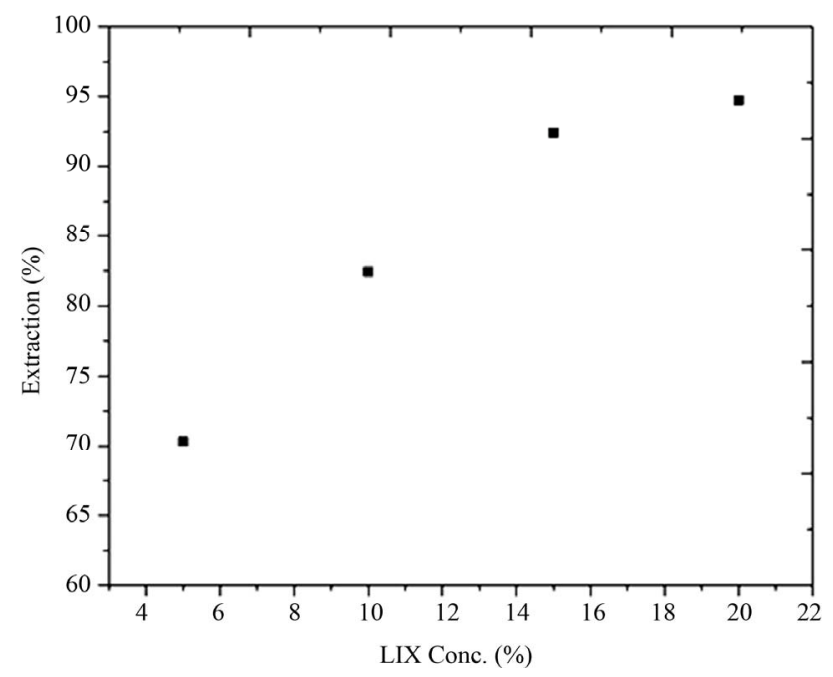

Figure 3. Effect of LIX 54 concentration on the extraction percent of Copper.

stripping agents for $\mathrm{Cu}$ recovery from organic phase. Table 1 showed that $0.1 \mathrm{M}$ sulfuric acid is the most suitable stripper for recovery of copper from organic phase.

\subsection{LEM Stability}

Surfactants play an important role in the formation and stability of liquid emulsion membrane. Different surfactants were tested for investigation the stability of prepared membrane. The leakage percent $(\% \mathrm{~B})$ of the internal aqueous phase to the external aqueous phase due to membrane break-up was obtained by plotting \%B against time $t$ as shown in Figure 4 Considerable leakage was observed when the emulsion was dispersed at the begining the extraction when using span 85 and span 80 , and $\% \mathrm{~B}$ increased with time $[16,17]$. The results revealed that sorbitan sesquioleate is the best stabilizing agent for prepared LEM. The effect of surfactant concentrations (sorbitan sesquioleate) in the prepared LEM were studied using different sorbitan sesquioleate concentrations varies from $4 \%$ to $12 \%$. Figure 5 showed that the stability of LEM increases with increasing sorbitan sesquioleate concentration but there is no major stability difference between $8 \%$ and $12 \%$ therefore $8 \%$ sorbitan sesquioleate concentration is selected as an optimum surfactant concentration not only to avoid the LEM swelling but also to avoid the difficulty for breaking up the primary emulsion to recover the extracted $\mathrm{Cu}[18]$.

\subsection{Factors Affecting the Extraction of Copper Using LEM}

Based on the results obtained from liquid-liquid extraction of copper, and the stability of the liquid emulsion membrane studies, the emulsion composition used during the investigations of some important factors affecting the
Table 1. Effect of different stripping agents on the recovery of $\mathrm{Cu}$.

\begin{tabular}{cc}
\hline Stripping agents (0.1 M) & Stripping efficiency \% \\
\hline $\mathrm{HCl}$ & 93 \\
$\mathrm{NaOH}$ & 16 \\
$\mathrm{H}_{2} \mathrm{SO}_{4}$ & 97 \\
Oxalic acid & 13 \\
Sodium acetate & 8 \\
Sodium citrate & 1.5 \\
\hline
\end{tabular}

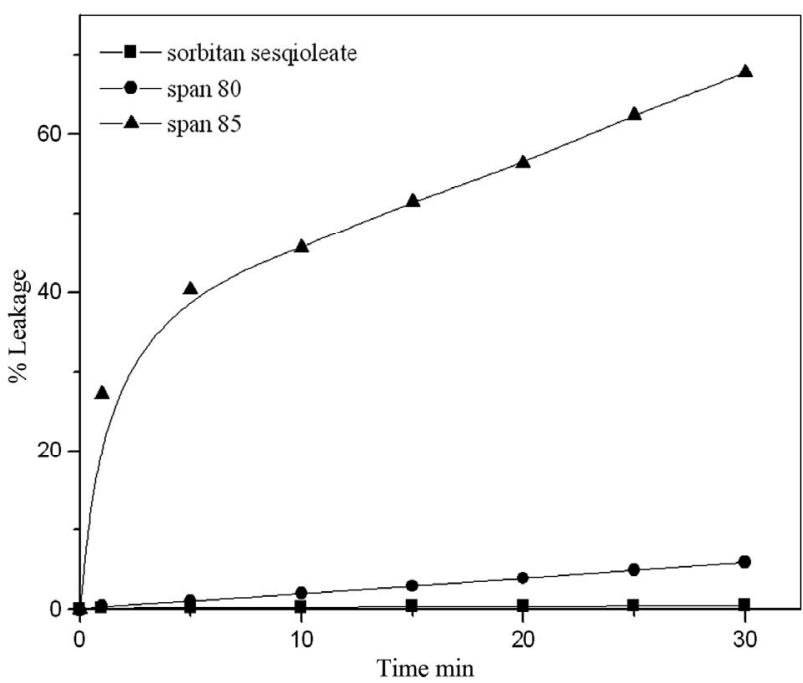

Figure 4. Stability of liquid emulsion membrane using different types of surfactant.

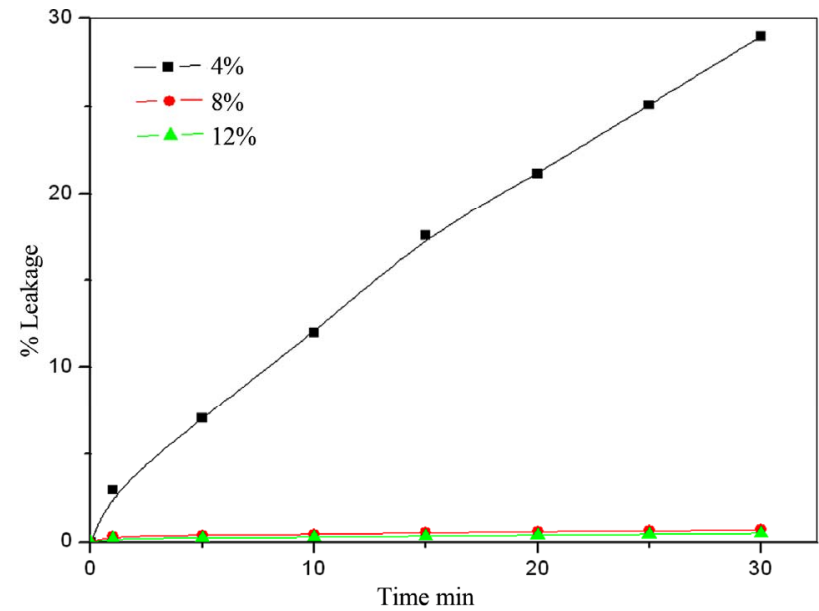

Figure 5. Stability of LEM with different sorbitan sesquioleate concentrations.

rate of permeation of copper through LEM is the following; unless otherwise stated: copper ion concentration in the external aqueous phase was $50 \mathrm{mg} / \mathrm{l}$ copper at $\mathrm{pH}=$ 4.3 , LIX 54 concentration was $(15 \%)(\mathrm{v} / \mathrm{v})$, nonionic surfactant sorbitan sesquioleate $8 \%(\mathrm{HLB}=3.7)(\mathrm{v} / \mathrm{v})$ in toluene, ratio of organic phase to internal aqueous phase was taken to be $1(\mathrm{v} / \mathrm{v})$, the emulsion phase volume to the 
external aqueous phase volume was $20: 100 \mathrm{v} / \mathrm{v}$, the temperature is $25^{\circ} \mathrm{C}$, the internal aqueous phase is $0.1 \mathrm{M}$ $\mathrm{H}_{2} \mathrm{SO}_{4}$, stirring speed is $450 \mathrm{rpm}$, emulsification speed $7000 \mathrm{rpm}$ and emulsification time is $5 \mathrm{~min}$.

\subsubsection{Effect of $\mathbf{p H}$ in the External Phase}

Figure 6 showed the effect of initial hydrogen ion concentrations of the external aqueous phase on the permeation \% of copper through LEM. Different $\mathrm{pHs}$ were used ranging from 1.0 to 5.0. It was found that, the permeation percent of copper increases with increasing $\mathrm{pH}$. This may be due to, the competition between positive charged ions which are copper and hydrogen ions at the membrane feed interface at high hydrogen ion concentration (low $\mathrm{pH}$ ).

\subsubsection{Effect of LIX 54 Concentration}

As shown in Figure 7, the effect of LIX 54 concentration on the permeation $\%$ of copper was studied using different

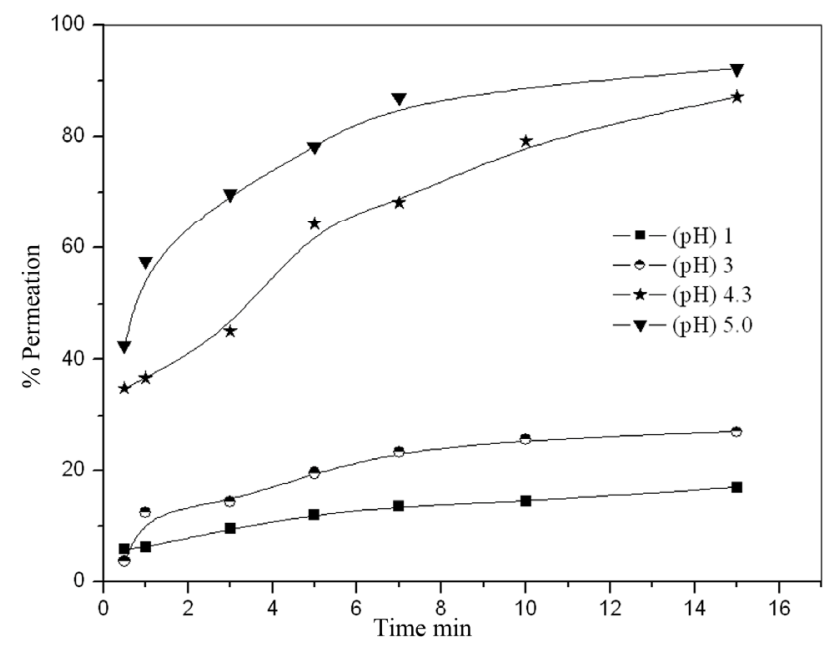

Figure 6. Effect of $\mathrm{pH}$ in external phase on the permeation $\%$ of copper.

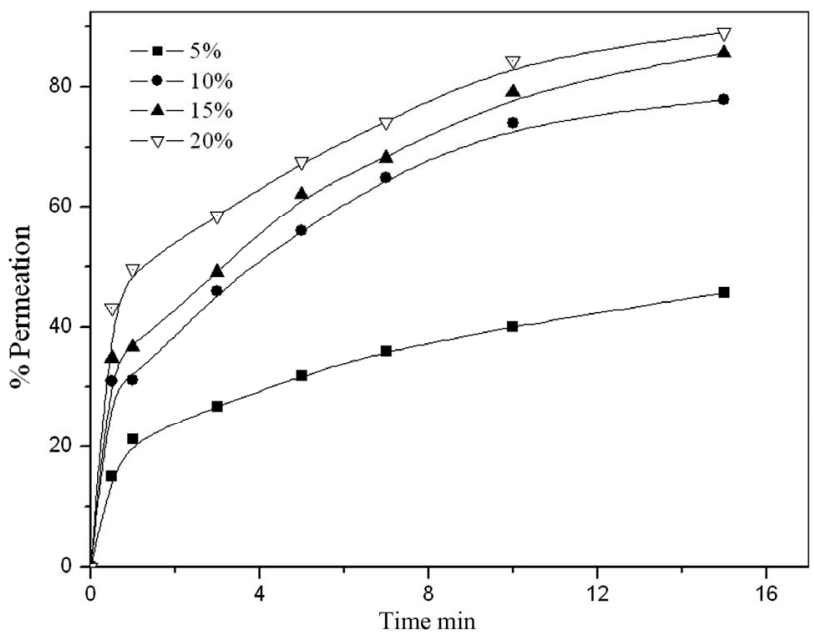

Figure 7. Effect of LIX-54 concentrations on the permeation $\%$ of copper. concentrations of LIX 54 from $5 \%$ to $20 \%$ in toluene. The results indicated that, the permeation $\%$ increases with increasing the carrier concentration (LIX 54), this is due to the fact that, with increasing LIX 54 concentration in the prepared LEM, the formation of LIX 54/copper complexes increased at the membrane/feed interface. Therefore the concentration gradient increases in the peripheral oil layer, which increases the diffusion rate of copper through LEM.

\subsubsection{Effect of Stirring Speed}

The effect of stirring speed on the permeation of copper through LEM is shown in Figure 8. Different stirring speeds were examined in the range 350 - $550 \mathrm{rpm}$. It was found that permeation \% of copper increases with increasing the stirring speed from 350 to $550 \mathrm{rpm}$. This is due to the fact that with increasing stirring speed, the number of emulsion globules increases and drop diameter of LEM in the stirring tank decreases. This leads to increase the surface area of membrane phase therefore the mass transfer resistances in the diffusion double layer decreases in addition to the coalescence of the droplets is assumed to be negligible when the external aqueous phase solution is well stirred.

\subsubsection{Effect of Membrane to External Aqueous Phase Ratio}

The effect of membrane to external aqueous phase ratio on the permeation \% of copper was studied by changing the volume of membrane phase while keeping the volume of external aqueous phase constant as $100 \mathrm{ml}$. The LEM volumes were 10, 25, 35 and $50 \mathrm{ml}$. Figure 9, showed that the permeation \% of copper increases with increasing the volume of membrane phase. This is due to the fact that with increasing emulsion volume not only the number of globules and surface area of LEM increases

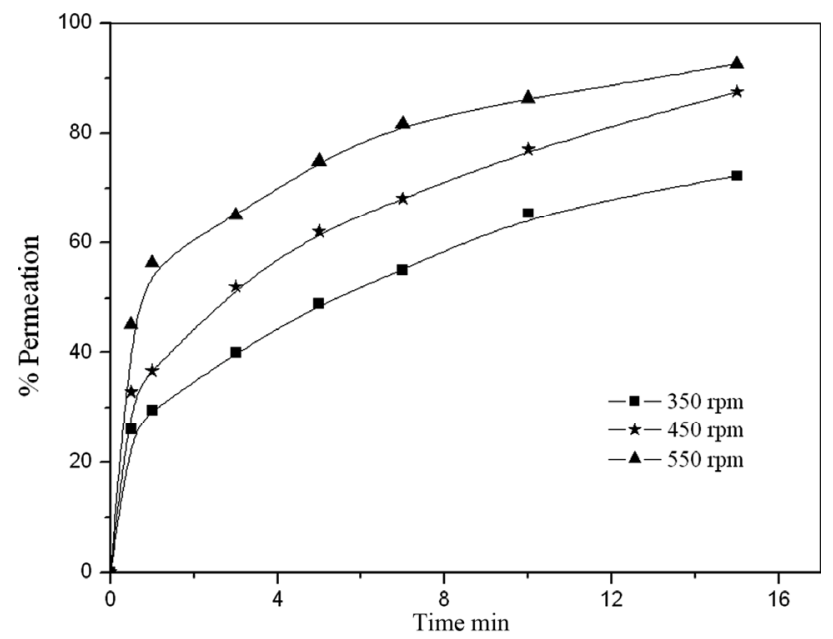

Figure 8. Effect of stirring speed on the permeation \% of copper. 


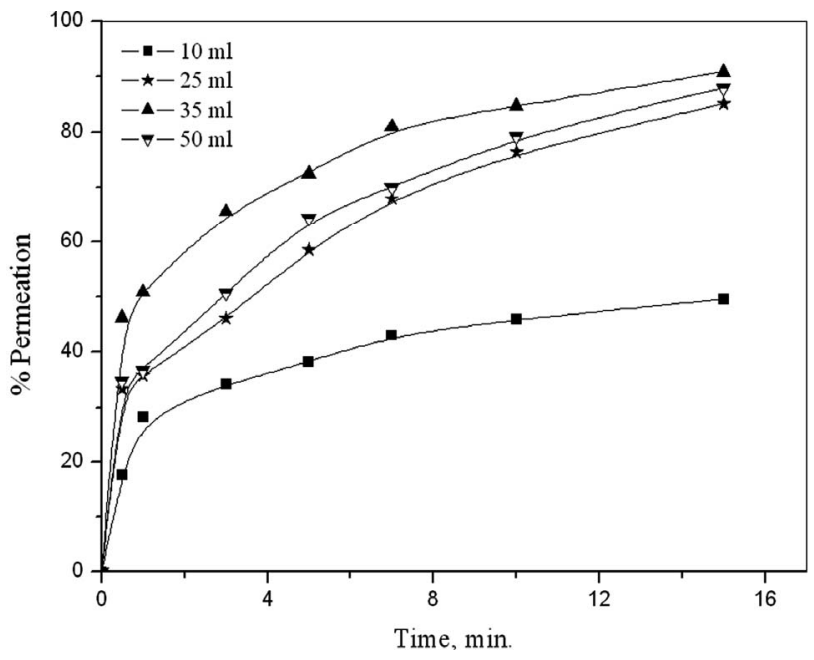

Figure 9. Effect of membrane to external aqueous phase ratio.

but also the number of active sites of LIX 54 on the membrane phase increase which lead to increase the total number of copper/LIX 54 complexes in the feed $/ \mathrm{mem}$ brane interface.

\subsubsection{Effect of Sulfuric Acid Concentration as Stripping Phase}

The results showed that the influence of sulfuric acid concentration as stripping agent on the extraction percent of copper is practically constant regardless of acid content in the stripping phase [19].

\section{Conclusion}

Copper removal from a waste stream by means of an emulsified liquid membrane has been studied. An experimental method was used, which allowed evaluation of the main variables. The liquid membrane was prepared by dissolving LIX-54 as a carrier and Sesquoleate as a surfactant. The membrane permitted a very efficient metal removal from the feed solution into the strip liquor, the experiments carried out in a batch-type stirred tank at $25^{\circ} \mathrm{C}$. from the experimental results, it was deduced that the statistically significant terms for copper transport through the membrane are the membrane stability, extractant concentration, stirring speed of the double emulsion and membrane ratio. However, it is not possible to increase the extractant concentration in the preparation of membrane to unlimited way since an increase in the absolute viscosity of the organic solvent occurs, producing a lower diffusion speed of the species, which affects the metal mass transfer process. The higher the stirring speed of the double emulsion, the smaller the size of the emulsion droplets, therefore increases the interface area for the extractant/metal reaction. Nevertheless, a maximum limit to raise this variable must be considered. An excessively high stirring speed produced coalescence, and finally breakdown of globules, turning the primary emulsion unstable. The concentration range of surfactant used in this experimental method produced an efficiently stabilized membrane. However, it did not have an effect on the metal transport. The use of an unnecessarily high content of the surfactant produces lower metal extraction due to the generation of higher interfacial resistance. The feasibility of removing low contents of metal in the extractor, using an emulsion liquid membrane, has been demonstrated in this work.

\section{REFERENCES}

[1] N. N. Li, Separating Hydrocarbons with Liquid Membranes, United States Patent 3410794, 1968.

[2] J. W. Franhenfeld, N. N. Li, "Wastewater Treatment by Liquid Ion Exchange in Liquid Membrane System," In: N. N. Li, Ed., Recent Developments in Separation Science, Vol. 3, CRC Press, Boca Raton, 1977. pp. 285-292.

[3] R. Marr and A. Kopp, "Liquid Membrane TechnologyA Survey of Phenomena, Mechanisms, and Models," International Chemical Engineering, Vol. 22, 1982, pp. 4460 .

[4] W. S. W. Ho and N. N. Li, "Recent Advances in Emulsion Liquid Membranes," In: R. A. Bartsch and W. J. Douglas, Eds., Chemical Separations with Liquid Membranes, ACS Symposium Series, Vol. 642, American Chemical Society, Washington DC, 1996, pp. 208-221.

[5] T. Kitagawa, Y. Nishikawa, J. W. Franhenfeld and N. N. Li, "Wastewater Treatment by Liquid Membrane Process," Environmental Science and Technology, Vol. 11, No. 6, 1977, pp. 602-605. http://dx.doi.org/10.1021/es60129a008

[6] B. A. Mikucki and K. Osseo-Asare, "The Liquid Surfactant Membrane Process: Effect of the Emulsifier Type on Copper Extraction by LIX65 N-LIX 63 Mixtures," Hydrometallurgy, Vol. 16, No. 2, 1986, pp. 209-229. http://dx.doi.org/10.1016/0304-386X(86)90044-7

[7] T. P. Martin and G. A. Davies, "The Extraction of Copper from Dilute Aqueous Solurtions Using a Liquid Membrane Process," Hydrometallurgy, Vol. 2, No. 4, 1977, pp. 315- 334.

http://dx.doi.org/10.1016/0304-386X(77)90040-8

[8] W. Volkel, W. Halwachs and K. Schugerl, "Copper Extraction by Means of a Liquid Surfant Memberane Process," Journal of Membrane Science, Vol. 6, 1980, pp. 1931. http://dx.doi.org/10.1016/S0376-7388(00)82146-8

[9] J. W. Franhenfeld, R. P. Chan and N. N. Li, "Extraction of Copper by Liquid Membranes," Separation Science and Technology, Vol. 16, No. 4, pp. 385-402. http://dx.doi.org/10.1080/01496398108068528

[10] S. B. Hu and J. M. Wiencek, "Emulsion liquid Membrane Extraction of Copper Using a Hollow-Fiber Contactor," AIChE Journal, Vol. 44, No. 3, 1998, pp. 570-581. http://dx.doi.org/10.1002/aic.690440308

[11] A. K. Chakravarti, S. B. Chowdhury and D. C. Mukherjee, 
"Liquid Membrane Multiple Emulsion Process of Separation of Copper $(\pi)$ from Wastewaters," Colloids and Surfaces, Vol. A166, 2000, pp. 7-25.

[12] T. Tsutomu, et al., "Extraction Equilibrium and Membrane Transport of Copper (II) with New N-6-(t-Dodecylamido)-2-Pyridinecarboxylic Acid in Polymer Inclusion Membrane," Industrial \& Engineering Chemistry Research, Vol. 46, No. 17, 2007, pp. 5715-5722. http://dx.doi.org/10.1021/ie061671u

[13] F. Valenzuela, J. Cabrera, C. Basualto and J. Sapag-Hagar, "Kinetics of Copper Removal from Acidic Mine Drainage by a Liquid Emulsion Membrane," Minerals Engineering, Vol. 18, No. 13-14, 2005, pp. 1224-1232.

http://dx.doi.org/10.1016/j.mineng.2005.07.015

[14] B. Sengupta, R. Sengupta and N. Subrahmanyam, "Copper Extraction into Emulsion Liquid Membranes Using LIX 984N-C R," Hudrometallurgy, Vol. 81, No. 1, 2006, pp. 67-73.

[15] M. Teresa and M. Jorge, "Modelling of Zinc Extraction from Sulphate Solutions with Bis(2-ethylhexyl)thiophosphoric Acid by Emulsion Liquid Membranes," Journal of Membrane Science, Vol. 237, No. 1-2, 2004, pp. 97-
107. http://dx.doi.org/10.1016/j.memsci.2004.02.025

[16] M. Djenouhat, O. Hamdaoui, M. Chiha and M. H. Samar, "Ultrasonication-Assisted Preparation of Water-in-Oil Emulsions and Application to the Removal of Cationic Dyes from Water by Emulsion Liquid Membrane: Part 1: Membrane Stability," Separation and Purification Technology, Vol. 62, No. 3, 2008, pp. 636-641

[17] O. Bechiri, F. Ismail, M. Abbessi and M. H. Samar, "Stability of the Emulsion (W/O): Application to the Extraction of a Dawson Type Heteropolyanion Complex in Aqueous Solution," Journal of Hazardous Materials, Vol. 152, No. 3, 2008, pp. 895-902. http://dx.doi.org/10.1016/j.jhazmat.2007.11.067

[18] S. Mohanty, "Emulsion Liquid Membranes: Applications in Separation Processes," Chemical Engineering World, Vol. 39, No. 1, pp. 36-40.

[19] F. Valenzuela, C. Fonseca, C. Basualto, O. Correa, C. Tapia and J. Sapag, "Removal of Copper Ions from a Waste Mine Water by a Liquid Emulsion Membrane Method," Mineral Engineering, Vol. 18, No. 1, 2005, pp. 3340. http://dx.doi.org/10.1016/j.mineng.2004.05.011 